\title{
Redox metabolism abnormalities in autistic children associated with mitochondrial disease
}

\author{
RE Frye ${ }^{1,2}$, R DeLaTorre ${ }^{3}$, H Taylor $^{4}$, J Slattery ${ }^{1,2}$, S Melnyk ${ }^{1,2}$, N Chowdhury ${ }^{1}$ and SJ James ${ }^{1,2}$
}

Research studies have uncovered several metabolic abnormalities associated with autism spectrum disorder (ASD), including mitochondrial disease (MD) and abnormal redox metabolism. Despite the close connection between mitochondrial dysfunction and oxidative stress, the relation between MD and oxidative stress in children with ASD has not been studied. Plasma markers of oxidative stress and measures of cognitive and language development and ASD behavior were obtained from 18 children diagnosed with ASD who met criteria for probable or definite MD per the Morava et al. criteria (ASD/MD) and 18 age and gender-matched ASD children without any biological markers or symptoms of MD (ASD/NoMD). Plasma measures of redox metabolism included reduced free glutathione (fGSH), oxidized glutathione (GSSG), the fGSH/GSSG ratio and 3-nitrotyrosine (3NT). In addition, a plasma measure of chronic immune activation, 3-chlorotyrosine (3CT), was also measured. Language was measured using the preschool language scale or the expressive one-word vocabulary test (depending on the age), adaptive behaviour was measured using the Vineland Adaptive Behavior Scale (VABS) and core autism symptoms were measured using the Autism Symptoms Questionnaire and the Social Responsiveness Scale. Children with ASD/MD were found to have lower scores on the communication and daily living skill subscales of the VABS despite having similar language and ASD symptoms. Children with ASD/MD demonstrated significantly higher levels of fGSH/GSSG and lower levels of GSSG as compared with children with ASD/NoMD, suggesting an overall more favourable glutathione redox status in the ASD/MD group. However, compare with controls, both ASD groups demonstrated lower fGSH and fGSH/GSSG, demonstrating that both groups suffer from redox abnormalities. Younger ASD/MD children had higher levels of 3CT than younger ASD/NoMD children because of an agerelated effect in the ASD/MD group. Both ASD groups demonstrated significantly higher $3 C T$ levels than control subjects, suggesting that chronic inflammation was present in both groups of children with ASD. Interestingly, 3NT was found to correlate positively with several measures of cognitive function, development and behavior for the ASD/MD group, but not the ASD/NoMD group, such that higher 3NT concentrations were associated with more favourable adaptive behaviour, language and ASDrelated behavior. To determine whether difference in receiving medications and/or supplements could account for the differences in redox and inflammatory biomarkers across ASD groups, we examined differences in medication and supplements across groups and their effect of redox and inflammatory biomarkers. Overall, significantly more participants in the ASD/MD group were receiving folate, vitamin B12, carnitine, co-enzyme Q10, B vitamins and antioxidants. We then determined whether folate, carnitine, co-enzyme Q10, B vitamins and/or antioxidants influenced redox or inflammatory biomarkers. Antioxidant supplementation was associated with a significantly lower GSSG, whereas antioxidants, co-enzyme Q10 and B vitamins were associated with a higher fGSH/GSSG ratio. There was no relation between folate, carnitine, co-enzyme Q10, B vitamins and antioxidants with $3 \mathrm{NT}, 3 \mathrm{CT}$ or fGSH. Overall, our findings suggest that ASD/MD children with a more chronic oxidized microenvironment have better development. We interpret this finding in light of the fact that more active mitochondrial can create a greater oxidized microenvironment especially when dysfunctional. Thus, compensatory upregulation of mitochondria which are dysfunctional may both increase activity and function at the expense of a more oxidized microenvironment. Although more ASD/MD children were receiving certain supplements, the use of such supplements were not found to be related to the redox biomarkers that were related to cognitive development or behavior in the ASD/MD group but could possibly account for the difference in glutathione metabolism noted between groups. This study suggests that different subgroups of children with ASD have different redox abnormalities, which may arise from different sources. A better understanding of the relationship between mitochondrial dysfunction in ASD and oxidative stress, along with other factors that may contribute to oxidative stress, will be critical to understanding how to guide treatment and management of ASD children. This study also suggests that it is important to identify ASD/MD children as they may respond differently to specific treatments because of their specific metabolic profile. Translational Psychiatry (2013) 3, e273; doi:10.1038/tp.2013.51; published online 18 June 2013

\footnotetext{
${ }^{1}$ Department of Pediatrics, University of Arkansas for Medical Sciences, Little Rock, AR, USA; ${ }^{2}$ Arkansas Children's Hospital Research Institute, Little Rock, AR, USA; ${ }^{3}$ Children's Learning Institute, University of Texas-Heath, Houston, TX, USA and ${ }^{4}$ Texas Institute for Rehabilitation Research, Houston, TX, USA Correspondence: Dr RE Frye, Department of Pediatrics, University of Arkansas for Medical Sciences, Slot 512-41B, Room R4041, 13 Children's Way, Little Rock, AR 72202, USA.

E-mail: REFrye@uams.edu

Keywords: autism; inflammation; endophenotypes; mitochondrial disease; oxidative stress

Received 15 January 2013; revised 22 April 2013; accepted 25 April 2013
} 


\section{Introduction}

The autism spectrum disorders (ASD) are a heterogeneous group of neurodevelopmental disorders that are defined by behavioral abnormalities, which specifically include impairments in communication and social interactions accompanied by restrictive and repetitive behaviors. ${ }^{1}$ An estimated 1 out of 88 individuals in the United States are currently affected with an ASD and the incidence is continuing to rise. ${ }^{2}$ Although several genetic syndromes, such as Fragile $X$ and Rett syndromes, have been associated with ASD, empirical studies have estimated that genetic syndromes only account for the minority of ASD cases. ${ }^{3}$ Furthermore, although many of the cognitive and behavioral features of ASD are thought to arise from dysfunction of the central nervous system, many fields of medicine have documented multiple physiological abnormalities associated with ASD that are central nervous system independent. ${ }^{4-8}$ Taken together, these reports suggest that ASD may arises from systemic, rather than organ specific, abnormalities, at least in some individuals. Over the last decade, research publications have started to implicate physiological systems that transcend organ-specific dysfunction, such as immune dysfunction, inflammation, impaired detoxification, redox regulation/oxidative stress and energy generation/mitochondrial dysfunction. ${ }^{8,9}$

ASD appears to be associated with systemic metabolic abnormalities, at least in a subset of individuals. ${ }^{8,10}$ We are just starting to learn about the nature and prevalence of these metabolic disorders in ASD. For example, the nature and prevalence of mitochondrial disease (MD) in ASD is still under investigation. A recent meta-analysis by Rossignol and Frye found that $5 \%$ of children with ASD meet criteria for a classic MD (ASD/MD) but that $30+\%$ of children in the general ASD population exhibit biomarkers consistent with MD. ${ }^{11}$ Recently, Frye demonstrated that up to $50 \%$ of children with ASD have biomarkers of MD that are consistently abnormal (that is, repeatable) and valid (that is, correlate with other biomarkers of MD). ${ }^{12}$ In another study, $80 \%$ of the children with ASD demonstrated lower than normal electron transport chain (ETC) function in lymphocytes when compared with typically developing controls. ${ }^{13}$ The discrepancies between the estimates of classic MD and biomarkers of MD in ASD may be attributed to several factors. Most studies that estimate MD prevalence in the general ASD population have used the modified Walker criterion to define MD. ${ }^{11,14}$ This criterion relies on significant reduction in ETC complex function and/or known mitochondrial DNA (mtDNA) mutations to diagnose MD. ${ }^{4,11}$ However, only $23 \%$ of children with ASD/MD have a known mtDNA mutation ${ }^{11}$ and some children with ASD/MD have ETC over-activity rather than ETC deficiencies. ${ }^{15,16}$ Furthermore, many ASD/MD cases do not manifest lactate elevation, a key biomarker commonly used to identify individuals with MD. ${ }^{11,13,15,17}$ This has raised the idea that children with ASD might have mitochondrial dysfunction that is more prevalent and distinct from classic MD.

The biological basis for mitochondrial dysfunction is unknown, but the fact that only $23 \%$ of children with ASD/ MD have a known mtDNA abnormality, ${ }^{4}$ suggests that MD may be acquired. Indeed, it has been suggested that systematic abnormalities in ASD, such as mitochondrial dysfunction, may arise from environmental triggers ${ }^{14}$ in genetically sensitive subpopulations. ${ }^{18,19}$ This notion is strongly supported by a recent study of dizygotic twins, which estimated that the environment contributed a greater percent of the risk of developing autistic disorder (55\%) as compared with genetic factors (37\%) with these factors contributing about equally for the broader ASD diagnosis. ${ }^{14}$ Mitochondria are central to this concept as polymorphisms in mitochondrial genes can result in susceptibility to disease ${ }^{20,21}$ and mitochondrial dysfunction can result from environmental exposures that have been implicated in the development of ASD such as heavy metals, ${ }^{22-25}$ chemicals, ${ }^{26}$ polychlorinated biphenyls $^{27}$ or pesticides ${ }^{28,29}$ as well as physiological abnormalities associated with ASD such as elevated tumor necrosis factor $\alpha,{ }^{30-32}$ glutathione deficiency ${ }^{33}$ and oxidative stress. $^{34}$

Oxidative stress may be a key mechanism by which mitochondria are negatively influenced by factors linked to ASD such as pro-oxidant environmental toxicants ${ }^{22-29}$ and immune abnormalities, ${ }^{8}$ as reactive oxygen species (ROS) can result in mitochondrial dysfunction. ${ }^{4,11}$ Further, dysfunctional mitochondria can create a self-perpetuating cycle of progressive damage that amplifies functional deficits. Indeed, mitochondria are a major source of ROS as well as the target for ROS-mediated damage. ETC complex I dysfunction can produce high levels of ROS and reactive nitrogen species (RNS). ${ }^{35,36}$ In turn, ROS and RNS can inhibit ETC function, such as complex I activity, ${ }^{37}$ causing further mitochondrial dysfunction. ${ }^{38}$ Thus, in this manner exogenous or endogenous sources of ROS can initiate or promote mitochondrial dysfunction and damage, especially if the mitochondria are genetically susceptible to dysfunction.

In addition to mitochondrial dysfunction, abnormalities in redox metabolism and oxidative stress appear to be major metabolic abnormalities that impact a significant portion of children with ASD. ${ }^{39-42}$ Four independent case-control studies from our group have documented glutathione deficits in plasma, immune cells and post-mortem brain from ASD children, ${ }^{6,43-45}$ but other groups have also verified glutathione abnormalities in post-mortem brain, ${ }^{46}$ demonstrated ROSmediated damage to $\mathrm{mtDNA}^{47}$ and lipids, ${ }^{48}$ identified abnormalities in other ROS biomarkers such as homocysteine ${ }^{49}$ as well as found epigenetic changes potentially related to redox abnormalities. ${ }^{50,51} \mathrm{~A}$ deficit in glutathione antioxidant capacity is well known to promote oxidative stress and increase vulnerability to pro-oxidant environmental exposures and oxidative damage. ${ }^{52,53}$ In addition, 3-nitrotyrosine (3NT), a biomarker of oxidative damage to proteins, and 3-chlorotyrosine (3CT), a biomarker of chronic inflammation, have been found to be elevated in plasma and brain of children with ASD. ${ }^{44,45}$

The interaction between redox homeostasis, inflammation and mitochondrial function is vital to maintaining healthy cell physiology and may be important in disease initiation, progression and treatment. One important unanswered question regarding these metabolic abnormalities is whether one of these metabolic abnormalities is a primary cause of the other abnormalities or whether these metabolic abnormalities constitute different subgroups of children with ASD. For example, as mitochondrial dysfunction is believed to increase 
oxidative stress, it could be hypothesized that ASD/MD children may be the same subgroup of children with ASD and redox abnormalities because their dysfunctional mitochondria are producing increased ROS/RNS. Alternatively, it may be that factors such as environmental toxins induce oxidative stress, which damages the mitochondria and result in MD. Furthermore, it may be that groups of children with ASD and various metabolic abnormalities do not overlap and are purely separate subgroups of children with ASD.

In this study, we begin to tease out the potential differences between these subgroups of ASD children with various metabolic abnormalities. To this end, two groups of children with ASD matched on age and gender were studied, those with ASD/MD and those in which MD was ruled out (ASD/ NoMD). Measures of redox metabolism and inflammation as well as measures of language, development and behavior were evaluated in these groups. Overall, this study demonstrates that children with ASD/MD have distinct abnormalities in redox metabolism, including a unique relationship between redox metabolism and language, development and behavior, as compared with children with ASD/NoMD. Taken together, these findings suggest that there are distinct subgroups and multiple reasons for abnormalities in redox metabolism in children with ASD.

\section{Materials and methods}

Participants. Study participants were recruited from patients seen in the University of Texas Medically Based Autism Clinic in the Department of Pediatrics at the University of Texas Health Science Center at Houston by the first author (REF) from 2008 to 2011. Children had been previously diagnosed by a developmental pediatrician, pediatric neurologist and/or clinical psychologist with an ASD, including Autistic Disorder, Asperger's Disorder or Pervasive Developmental Disorder Not Otherwise Specified. An ASD was confirmed using Diagnostic Statistical ManualVersion IV-Text Revision diagnostic criteria at the time of evaluation using a standard neurodevelopment examination and interview. In addition, all other relatively common genetic or neurological conditions that could mimic ASD were ruled out. Parents of patients were requested to consent to allow their child's medical information to be anonymously abstracted into a clinical database that contained medical history, physical examination findings and the results of neurological and metabolic testing. Approximately $98 \%$ of parents (326 total patients) signed the consent.

A standardized metabolic diagnostic workup for disorders of mitochondrial metabolism disorders was conducted on most patients. ${ }^{4,11,54,55}$ The algorithm for this evaluation is depicted in Figure 1. Initial testing included laboratory tests to investigate abnormalities in respiratory chain, tricarboxylic acid cycle and fatty acid oxidation pathways. Abnormalities detected in initial testing were confirmed with repeat testing. If abnormalities could not be replicated on repeat testing, laboratory tests were reconsidered during metabolic stress or illness if the index of suspicion for MD remained high for the patient.

If MD was suspected, mtDNA examination was conducted to identify abnormalities by either a targeted analysis for common mutations and/or deletions and/or sequencing of the entire mtDNA genome (Baylor Medical Genetics Laboratory, Houston, TX, USA). ${ }^{56,57}$ When a conclusive mtDNA abnormality could not be identified, nuclear mitochondrial gene testing and/or a muscle and/or skin biopsy was recommended.

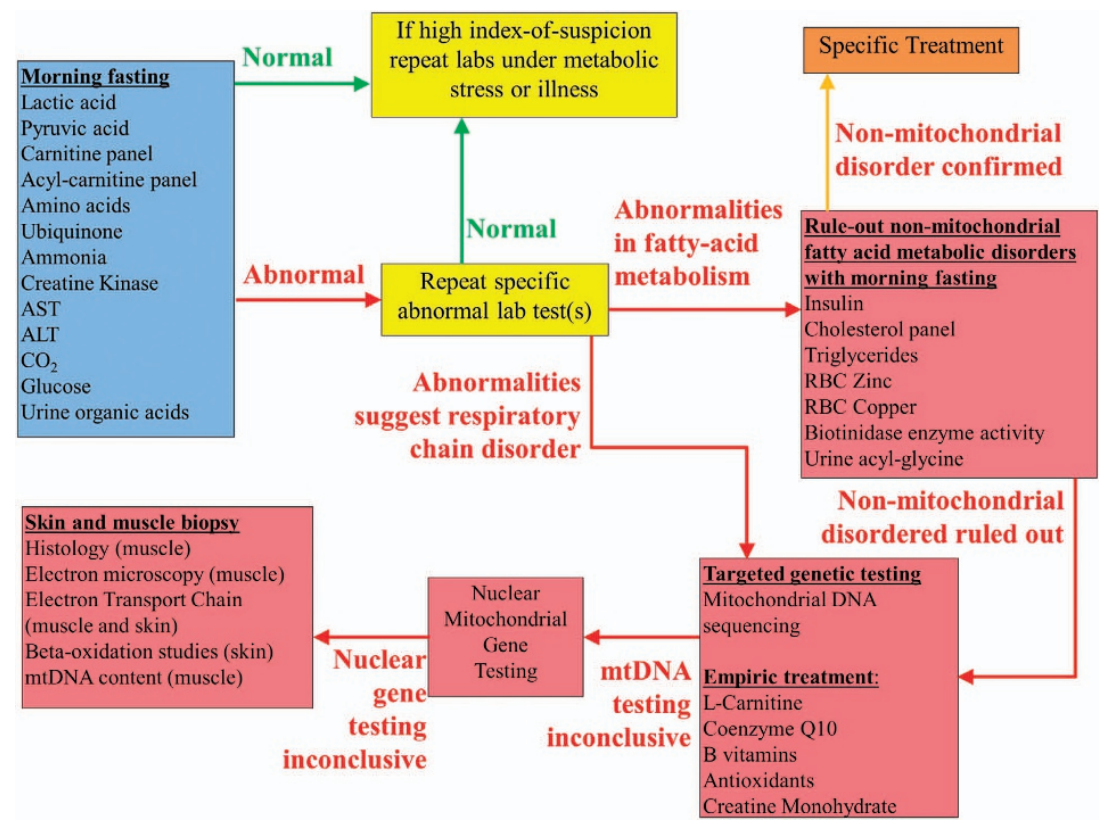

Figure 1 Algorithm for metabolic workup for mitochondrial disease in autistic spectrum disease patients. Patients are screened with biomarkers of abnormal mitochondrial function in the fasting state. Abnormalities are verified with repeat fasting biomarker testing. For patients with biomarkers for a fatty acid oxidation defect, other disorders of fatty acid metabolism are ruled out before further workup for a mitochondrial disease. Patients with consistent biomarkers for mitochondrial disease are first investigated for genetic causes of their mitochondrial disorder before considering a muscle and/or skin biopsy. ALT, alanine aminotranferease; AST, aspartate aminotransferase; mtDNA, mitochondrial DNA; RBC, red blood cell. 
Nuclear mitochondrial gene abnormalities were ruled-out in selected patients using an oligonucleotide array comparative genomic hybridization analysis that examines approximately 180 nuclear genes involved in mitochondria function, including genes involved in fatty acid oxidation, carnitine metabolism, biogenesis, mtDNA maintenance, transcription and translation or ETC complex assembly (MitoMet, Baylor Medical Genetics Laboratory). If no genetic abnormalities could be identified, a muscle and/or skin biopsy was recommended. Muscle was analyzed with light and electron microscopy as well as mtDNA content. ${ }^{58,59}$ Fibroblasts from the skin biopsy were cultured for functional fatty acid oxidation pathway testing. ETC function was examined on fresh frozen muscle and cultured fibroblasts (Baylor Medical Genetics Laboratory). ${ }^{60}$ Fibroblasts were incubated with d3-palmitate and $\mathrm{L}$-carnitine in duplicate for $72 \mathrm{~h}$ to determine the function of the beta-oxidation pathway (Baylor Institute of Metabolic Disease, Dallas, TX, USA). ${ }^{61}$

Because of issues with the sensitivity of the modified Walker criteria for the diagnosis of MD in ASD (as outlined in the introduction), the Morava et al. criterion, ${ }^{62}$ another widely used criteria, was used to diagnose patients with MD, as previously suggested. ${ }^{4}$ This criterion recognizes several types of clinical presentations, including primary muscular or central nervous system presentations or multisystem presentations, and metabolic, imaging and morphology data. The likelihood of a MD is determined by adding up the number of points associated with specific symptoms to get a MD criteria score. The MD criteria score rates MD as not likely $(\leqslant 1)$, possible ( $2-4$ points), probable $(5-7$ points) or definite $(\geqslant 8$ points). The MD criteria score and the points for the various presentations and laboratory findings for each ASD/MD participant are provided in Table 1.

Patients diagnosed with ASD/MD were recruited for this study. Age- and gender-matched patients who had undergone the MD screening portion of the workup (that is, biomarker testing) but were found to have no abnormal biomarkers on any testing were recruited to be ASD/NoMD participants. Patients were excluded if they had a genetic syndrome such as Fragile $\mathrm{X}$, Rett syndrome, Down syndrome or Angelman syndrome or prematurity. A total of 18 ASD/MD (age mean 8.5 years, s.d. 3.0 years) and 18 ASD/NoMD (age mean 7.9 years, s.d. 3.2 years) patients participated in the study. The participants were matched on age within an average of 8.8 months of age (s.d. 6 months). The ASD/MD group contained 14 males and 4 females, whereas the ASD/NoMD group contained 15 males and 3 females; there was one gender mismatch.

Cognitive and behavioral evaluations. All cognitive evaluations were conducted or supervised by the third author, a child psychologist (HT). The assessment included measures of adaptive behavior and language abilities. Parents completed rating scales that assessed autism symptoms and social reciprocity skills.

Adaptive behavior was assessed using the Vineland Adaptive Behavior Scale-Second Edition (VABS; Pearson

Table 1 Mitochondrial disease criteria score for 18 participants who met criteria for probable or definite mitochondrial disease

\begin{tabular}{|c|c|c|c|c|c|c|c|c|}
\hline Subject & Muscular & $\begin{array}{r}\text { Nervous } \\
\text { System }\end{array}$ & $\begin{array}{l}\text { Multi- } \\
\text { system }\end{array}$ & $\begin{array}{r}\text { Total } \\
\text { Clinical }\end{array}$ & $\begin{array}{l}\text { Metabolic } \\
\text { \& Imaging }\end{array}$ & $\begin{array}{r}\text { Muscle } \\
\text { Morphology }\end{array}$ & $\begin{array}{r}\text { Total } \\
\text { Points }\end{array}$ & $\begin{array}{l}\text { Probability of } \\
\text { Mitochondrial } \\
\text { Disease }\end{array}$ \\
\hline 1 & 0 & 2 & 2 & 4 & 2 & 0 & 6 & Probable \\
\hline 2 & 0 & 2 & 2 & 4 & 1 & 2 & 7 & Probable \\
\hline 3 & 0 & 2 & 1 & 3 & 1 & 4 & 9 & Definite \\
\hline 4 & 0 & 2 & 0 & 2 & 2 & 2 & 6 & Probable \\
\hline 5 & 1 & 1 & 1 & 3 & 4 & 2 & 9 & Definite \\
\hline 6 & 0 & 2 & 2 & 4 & 4 & 4 & 12 & Definite \\
\hline 7 & 0 & 2 & 2 & 4 & 4 & 2 & 10 & Definite \\
\hline 8 & 2 & 1 & 1 & 4 & 0 & 2 & 6 & Probable \\
\hline 9 & 0 & 2 & 2 & 4 & 2 & 2 & 8 & Definite \\
\hline 10 & 0 & 1 & 1 & 2 & 2 & 2 & 6 & Probable \\
\hline 11 & 0 & 2 & 3 & 5 & 0 & 2 & 6 & Probable \\
\hline 12 & 0 & 1 & 2 & 3 & 4 & 2 & 9 & Definite \\
\hline 13 & 0 & 2 & 2 & 4 & 4 & 0 & 8 & Definite \\
\hline 14 & 0 & 2 & 1 & 3 & 4 & 2 & 9 & Definite \\
\hline 15 & 0 & 1 & 1 & 2 & 4 & 2 & 8 & Definite \\
\hline 16 & 0 & 3 & 4 & 7 & 2 & 0 & 6 & Probable \\
\hline$\overline{17}$ & 0 & 2 & 2 & 4 & 4 & 0 & 8 & Definite \\
\hline 18 & 0 & 2 & 1 & 3 & 4 & 2 & 12 & Definite \\
\hline
\end{tabular}

The total score for each category of the Morava et al. criteria is outlined. However, the total points are calculated with regard to the maximum allowed score for each category so the total points, in some cases, may not add up to the sum of all of the categories. 
Education, San Antonio, TX, USA). ${ }^{63}$ The VABS is a semistructured interview designed to assess communication, daily living and socialization skills. Scales representing these skills generate a scaled score, which is used in this study; higher scores indicate better performance.

Language skills were assessed using two different tools depending on the age of the child. If the child was less than 7 years of age, the Preschool Language Scale-Fourth Edition (PLS; Pearson Education, San Antonio, TX, USA $)^{64}$ was used. The PLS is a direct assessment of total, receptive and expressive language abilities in children aged birth to 6 years, 11 months. The PLS generates a total language score, which is represented as an age-equivalent scores for this study. Higher subscale scores indicate better skills. Normative values are based on a diverse sample of over 1500 children. For children 7 years and older, the Expressive One-Word Picture Vocabulary Test-Fourth Edition (EOWPVT; Academic Therapy Publications, Novato, CA, USA) was used. The EOWPVT assesses a participant's ability to name illustrations of objects, actions and concepts using one word. ${ }^{65}$ The normative values for the EOWPVT are based on a nationally representative sample of 2394 individuals age 2 to $80+$ years stratified by region, metropolitan area, ethnicity, gender and education level. The EOWPVT provides a total age-equivalent score, which was used in this study. To generate a measure to represent language delay that could be derived from either language test, the participant's age was subtracted from the age-equivalent score derived from the language test. This score represents the language delay in months.

Social skills were assessed using the social responsiveness scale (SRS). ${ }^{66}$ The SRS is a 65 -item scale used to measure the severity of ASD symptoms as they occur in natural social settings. The measure is used in children aged 4-18 years of age. The SRS is comprised of five subscales represented by standard $T$-scores based on gender. Higher subscale scores indicate worse symptoms. Subscales include social awareness, social cognition, social communication, social motivation and autism mannerisms.

Autism symptoms were assessed using the autism symptoms questionnaire (ASQ). The ASQ was developed by The Center for Autism and Related Disorders (Tarzana, CA, USA). It is a structured questionnaire of simple standardized yes/no questions that reviews Diagnostic and Statistical Manual of Mental Disorders-Fourth Edition-Text Revision symptoms of social interactions, communication and stereotyped behavior and provides a diagnosis of Autistic Disorder, Asperger's Disorder or Pervasive Developmental Disorder-Not Otherwise Specified or indicates that the symptoms are not consistent with an ASD diagnosis. The ASQ generates a score from 0 to 4 for the social interaction and stereotyped behavior scales and a score from 0 to 5 for the communication scale. Only the total score was used in the analysis in order to provide a measure of ASD symptoms with a large enough variation for a valid statistical analysis. Higher score represents more (worse) ASD symptoms.

Redox and inflammatory biomarkers. Free glutathione is synthesized in its reduced form ( $\mathrm{fGSH}$ ) and can be oxidized by reactive species to the oxidized disulfide form (GSSG). The free reduced-to-oxidized glutathione ratio (fGSH/GSSG) is an excellent indicator of redox metabolism. fGSH, GSSG and $\mathrm{fGSH} / \mathrm{GSSG}$ were measured in plasma and represent extracellular redox status. To examine an index of chronic effects of oxidative stress, a marker of protein damage secondary to reactive species, 3NT, was measured in plasma. Last, 3CT, a measure of chronic immune activation was also measured in plasma. All biomarkers were collected as morning fasting samples before the morning dose of any medication or supplement. Any vitamin supplement that could affect mitochondrial or redox metabolism were held for at least $24 \mathrm{~h}$ before the blood draw. Oxidative stress and inflammatory biomarkers included fGSH, GSSG, fGSH/ GSSG, 3NT and 3CT. At least $4 \mathrm{ml}$ of blood was collected into an EDTA-Vacutainer tube and immediately chilled on ice before centrifuging at $4000 \mathrm{~g}$ for $10 \mathrm{~min}$ at $4{ }^{\circ} \mathrm{C}$. To prevent metabolite interconversion, the ice-cold samples were centrifuged within $15 \mathrm{~min}$ of the blood collection and the plasma stored at $-80^{\circ} \mathrm{C}$ until high-performance liquid chromatography quantification within 2 weeks after receipt. Details of the methodology for high-performance liquid chromatography with electrochemical detection and metabolite quantitation have been previously described. ${ }^{67}$ The values of these markers in the ASD groups were compared with normative values established in a previous study on redox metabolism. ${ }^{44}$ These controls included 54 healthy children ranging from 3 to 10 years of age with no history of developmental delay or neurological symptoms.

Analytic approach. For most of the analyses, mixed-effect regression models ${ }^{68}$ were conducted via SAS version 9.3 (SAS, Cary, NC, USA) 'glmmix' procedure. Data were not transformed for analysis, all available data were used and there was no imputation of missing values. The mixed-effect models allowed each participant's data to be compared specifically to the match participant. The first set of analyses compared cognitive, oxidative stress and inflammatory biomarkers across groups using only a between-group dichotomous effect of ASD group. The second set of analyses determined whether there was any effect of age on the redox or inflammatory biomarkers and whether this effect was different across the two ASD groups. In this analysis, age was represented as a continuous variable with the between-group dichotomous effect of ASD group as well as the interaction between these variables. The relationship between cognitive/ behavioral variables and redox and inflammatory biomarkers were investigated. In this analysis, the biomarker was consider the response variable. The model contained a specific continuous cognitive/behavioral variable with the between-group dichotomous effect of ASD group as well as the interaction between these variables. When the effect of age and its interaction with ASD group was significant, these factors were included in the analysis. The number of participants receiving specific classes of medication and supplements were determined for each group and differences in frequency of receiving such supplements were compared across groups using a $\chi^{2}$. Differences in age of the participants across supplement use and ASD group membership were analyzed using the mixed-model as was an analysis of the difference in redox and inflammatory markers across medication/supplement status. For the mixed-model, 
F-tests were used to evaluate significance. T-tests were used to compare biomarkers between the each of the ASD groups to the controls groups. In general, $P$ values $<0.05$ were used to indicate statistical significance.

\section{Results}

Differences in development, language and behavior between ASD subgroups. As seen in Figure 2, the ASD/ MD group demonstrated significantly lower scores for communication $(\mathrm{F}(1,14)=7.06, P=0.02)$ and daily living skills $(\mathrm{F}(1,16)=9.65, P<0.01)$, and borderline lower scores for social skill $(F(1,16)=3.17, P<0.10)$ of the VABS but similar scores for language, autistic symptoms on the ASQ and social symptoms on the SRS.

Differences in redox and inflammatory biomarkers between ASD groups. As seen in Figure 3, plasma 3CT $(\mathrm{F}(1,16)=5.23, P<0.05)$ and $\mathrm{fGSH} / \mathrm{GSSG}(\mathrm{F}(1,16)=8.19$, $P=0.01)$ were higher in the ASD/MD group as compared with the ASD/NoMD group, whereas plasma GSSG $(\mathrm{F}(1,16)=5.90, \quad P<0.05)$ was higher in the ASD/NoMD group as compared with the ASD/MD group. The remaining plasma redox biomarkers were not significantly different between the two groups.

Differences in redox and inflammatory biomarkers between ASD groups and control children. As seen in Figure 3, compared with controls, both ASD/NoMD $(t(35)=7.26, \quad P<0.001) \quad$ and $\mathrm{ASD} / \mathrm{MD} \quad(t(35)=9.33$, $P<0.001)$ groups demonstrated significantly lower plasma $\mathrm{fGSH}$ levels. As compared with controls, the ASD/NoMD group $(t(35)=3.56, \quad P<0.001)$ but not ASD/MD group demonstrated significantly higher plasma GSSG levels. As compared with controls both ASD/NoMD $(t(35)=9.65$, $P<0.001)$ and ASD/MD $(t(35)=8.24, P<0.001)$ demonstrated significantly lower fGSH/GSSG ratios. Neither ASD/
NoMD nor ASD/MD groups demonstrated any differences in plasma 3NT as compared with the control group. As compared with controls, both ASD/NoMD $(t(35)=14.31$, $P<0.001)$ and ASD/MD $(t(35)=7.50, P<0.001)$ demonstrated significantly higher plasma 3 CT levels.

Relationship between age and inflammatory biomarker. Plasma 3CT decreased with age for the ASD/MD group but not for the ASD/NoMD group (age $\times$ group interaction, $\mathrm{F}(1,14)=5.19, P<0.05$; see Figure $4 a$ ). Plasma $3 \mathrm{CT}$ was still higher for the ASD/MD group as compared with the ASD/ NoMD group even when accounting for the effect of age $(\mathrm{F}(1,14)=8.95, P<0.01)$. Age did not affect any of the measures of oxidative stress.

Relationship between inflammatory biomarker and cognitive, language and autism symptoms. The relationship between plasma $3 \mathrm{CT}$ and the rating of autistic symptoms, as indexed by the ASQ, was different for the ASD/MD and ASD/ NoMD groups (interaction $\mathrm{F}(1,11)=8.20, P=0.01$ ). This was due to the fact that, for the ASD/MD group but not the ASD/NoMD group, the relationship between plasma 3CT and autism symptoms was significant $(F(1,14)=5.59, P<0.05)$. Fewer total autism symptoms (better function) was associated with higher plasma 3CT values for the ASD/MD group but not the ASD/NoMD group. However, as seen in Figure $4 \mathrm{~b}$, the relationship is somewhat variable and may be driven by a limited number of participants. Plasma 3CT was not found to be related to VABS, language or the SRS.

Relationship between age and redox biomarkers. Age was not related to any measure of redox metabolism.

Relationship between adaptive behavior and redox metabolism. The relationship between plasma 3NT and adaptive behavior was different for the ASD/MD and ASD/ NoMD groups for communication (interaction $F(1,14)=8.08$, $P=0.01$ ), daily living (interaction $\mathrm{F}(1,14)=7.72, P=0.01$ )
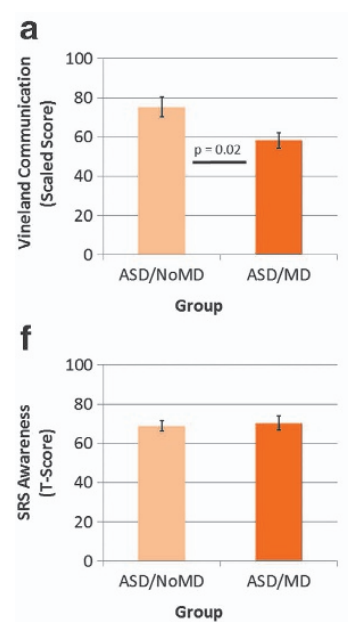

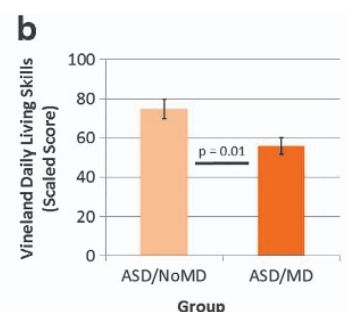

g

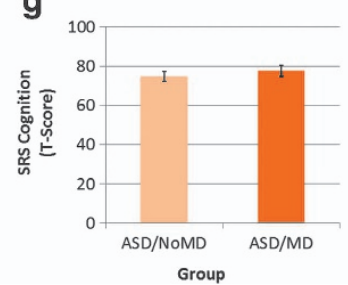

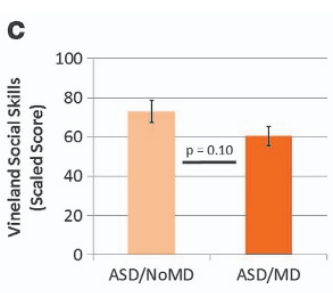

h

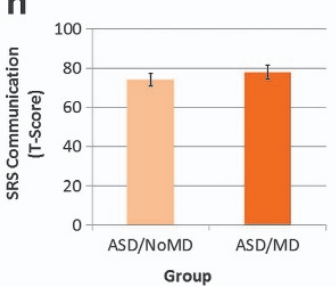

d

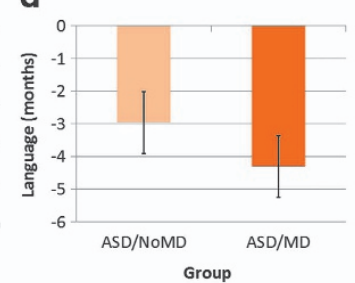

i

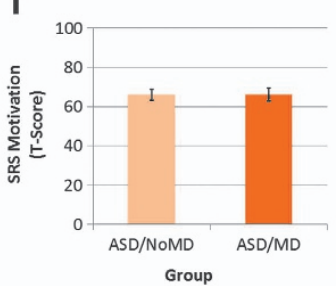

e

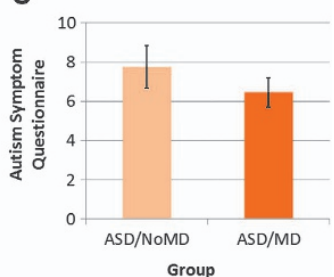

j

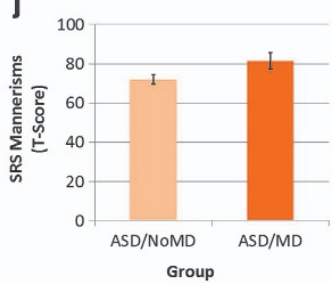

Figure 2 Cognitive and behavioral characteristics for two groups of children with autism spectrum disorder (ASD): those with mitochondrial disease (MD; ASD/MD) and those without mitochondrial disease (ASD/NoMD). Children with ASD/MD demonstrated lower scores on the Vineland Adaptive Behavior Scale-Second Edition (a) communication and (b) daily living skill subscales as compared with the ASD/NoMD children. Borderline significant differences were found between the two groups for (c) social skills as measured by the Vineland Adaptive Behavior Scale. No differences were found between the two groups on (d) language (as determined by the Preschool Language Scales or the Expressive One-Word Picture Vocabulary Test depending on the child's age), (e) the ASQ and (f-j) subscales of the Social Responsiveness Scale (SRS). 
a

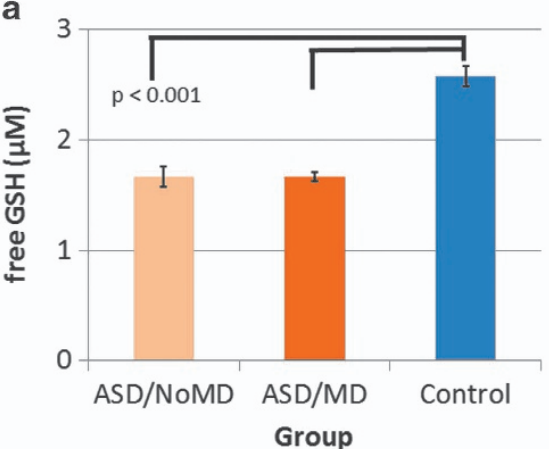

C
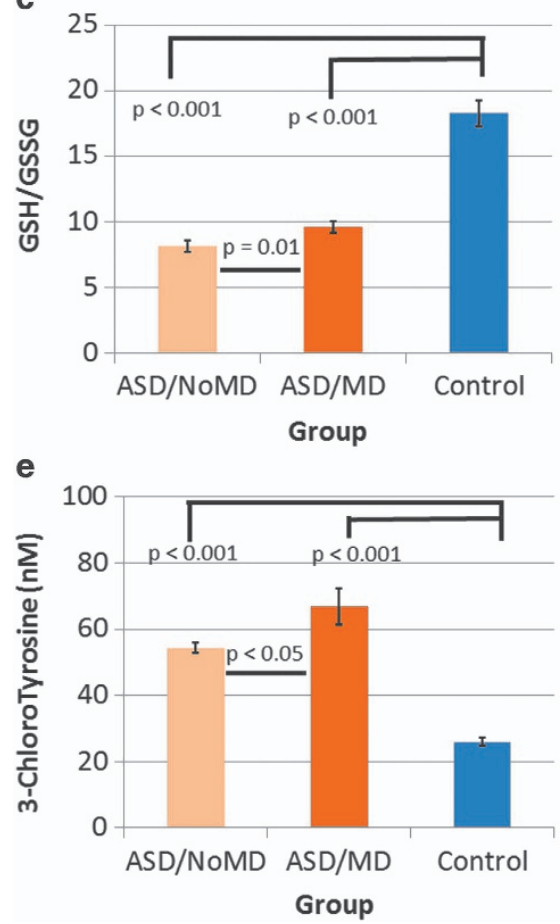

b

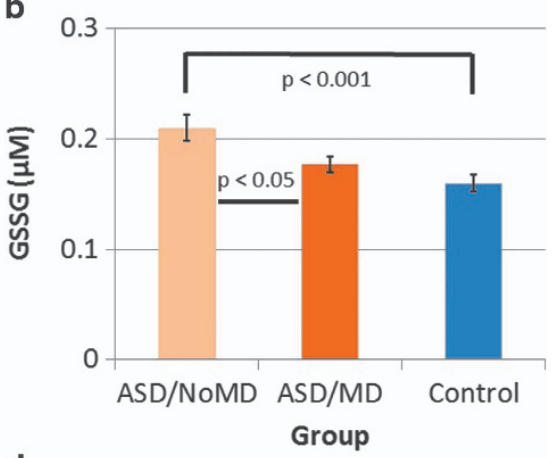

d

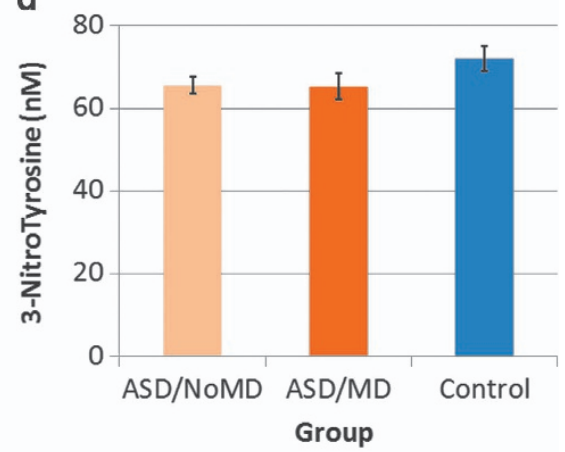

Figure 3 A comparisons of biomarkers of oxidative stress, including (a-c) glutathione metabolism (free reduced glutathione (fGSH), oxidized glutathione (GSSG), fGSH/ GSSG ratio) and (d) chronic protein oxidation (3-nitrotyrosine (3NT)), and (e) inflammation (3-chlorotyrosine (3CT)) between two groups of children with autism spectrum disorder (ASD), those with mitochondrial disease (ASD/MD) and those without mitochondrial disease (MD; ASD/NoMD), and typically developing controls.
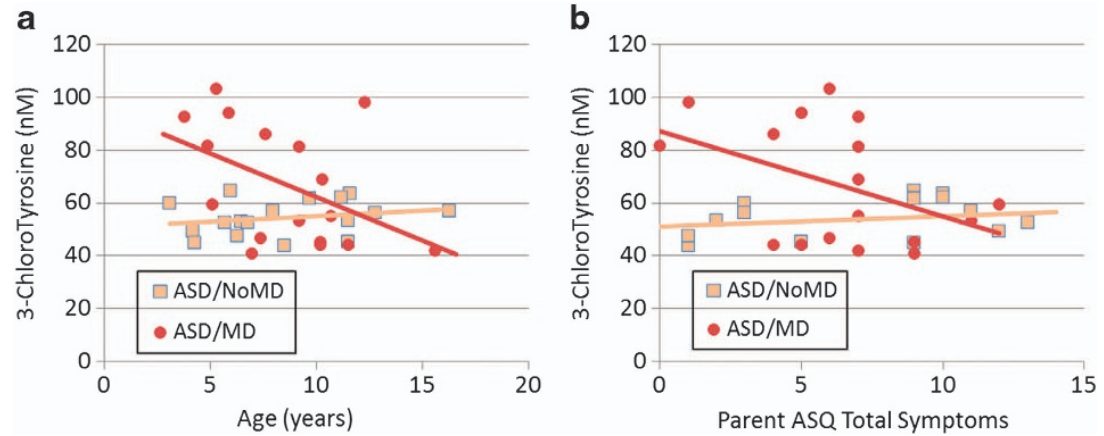

Figure 4 3-Chlorotyrosine, a marker of chronic inflammation, in two groups of children with autism spectrum disorder (ASD): those with mitochondrial disease (ASD/MD) and those without mitochondrial disease (MD; ASD/NoMD). (a) 3-Chlorotyrosine decreases with age for the ASD/MD but not the ASD/NoMD groups. (b) 3-Chlorotyrosine is related to parental ratings of autism symptoms for the ASD/MD but not the ASD/NoMD group. ASQ, Autism Symptoms Questionnaire.

and social (interaction $\mathrm{F}(1,14)=6.97, P=0.02$ ) skill subscales on the VABS. This was due to the fact that, for the ASD/MD group but not for the ASD/NoMD group, the relationship between plasma $3 \mathrm{NT}$ and communication $(\mathrm{F}(1,15)=10.29, \quad P<0.01)$, daily living $(\mathrm{F}(1,15)=9.21$, $P<0.01)$ and social $(F(1,15)=12.12, P<0.01)$ skills was 
a

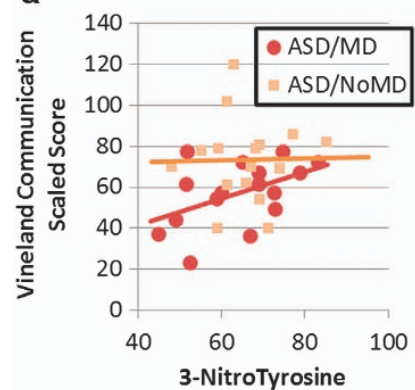

e

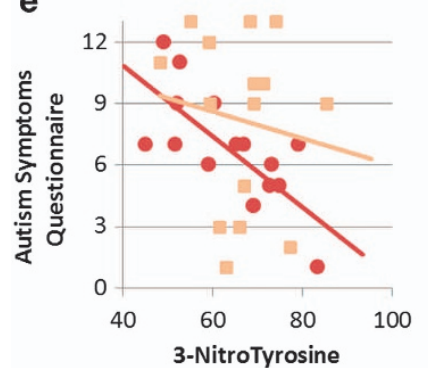

b

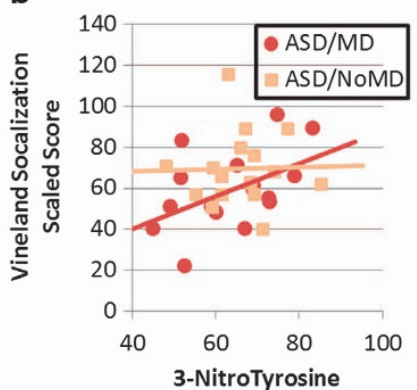

f

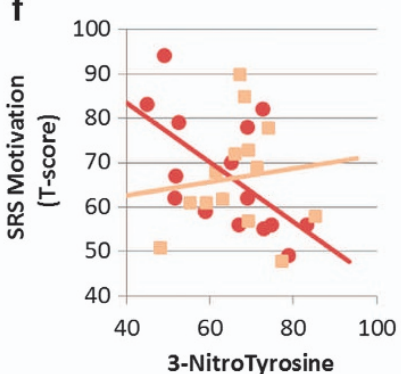

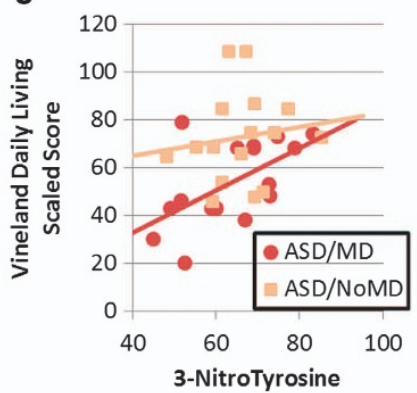

g

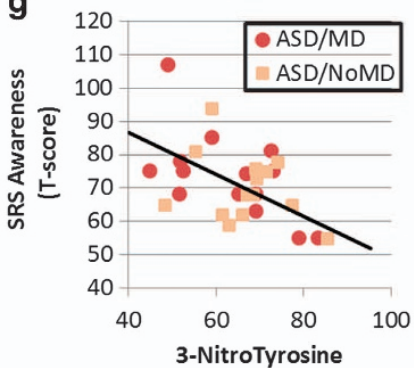

d

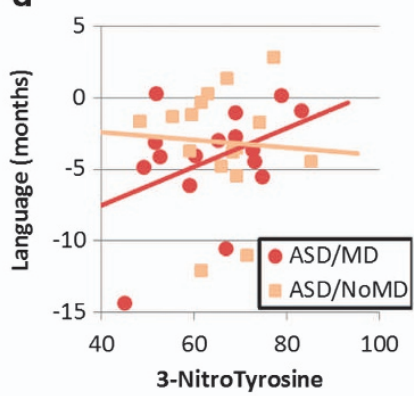

Figure 5 The relationship between 3-nitrotyrosine, a marker of chronic protein oxidation, and measures of cognition, language and autism symptoms in two groups of children with autism spectrum disorder (ASD): those with mitochondrial disease (MD; ASD/MD) and those without mitochondrial disease (ASD/NoMD). In general, concentrations of 3-nitrotyrosine were related to measures of cognitive development and autistic behavior in the ASD/MD but not the ASD/NoMD group. Specifically, more favorable performance in communication (a), socialization (b) and (c) daily living skills on the Vineland Adaptive Behavior Scale-Second Edition, (d) language (as determined by the Preschool Language Scales or the Expressive One-Word Picture Vocabulary Test depending on the child's age), (e) autism symptoms rated by the parents using the Autism Symptoms Questionnaire and (f) social motivation as rated by the parents using the Social Responsiveness Scale (SRS) were related to higher concentrations of 3-nitrotyrosine in the ASD/MD, but not the ASD/NoMD, group. (g) Interestingly, more favorable performance on the social motivation as rated by the parents using the SRS were related to higher concentrations of 3-nitrotyrosine in both the ASD/MD and ASD/NoMD groups.

significant. As seen in Figures $5 \mathrm{a}-\mathrm{c}$, for the ASD/MD group, performance on these subscales was higher (better function) for participants with higher plasma 3NT values for the ASD/ MD group but there was no relationship between plasma 3NT and performance for the ASD/NoMD group. No relationship was found for the subscales of the VABS and plasma glutathione biomarkers.

Relationship between language and redox metabolism. The relationship between plasma 3NT and language was different for the ASD/MD and ASD/NoMD groups (interaction $\mathrm{F}(1,14)=4.83, P=0.05)$. This was due to the fact that, for the ASD/MD group but not the ASD/NoMD group, the relationship between plasma 3NT and language $(F(1,15)=6.16, P<0.05)$ was significant. As seen in Figure $5 d$, language scores were higher (better language) for participants with higher plasma 3NT values for the ASD/MD group but there was no relationship between plasma $3 N T$ and language for the ASD/NoMD group. No relationship was found for language and plasma glutathione biomarkers.

Relationship between autism symptoms and redox biomarkers. The relationship between plasma 3NT and the rating of autistic symptoms was different for the ASD/MD and ASD/NoMD groups (interaction $F(1,13)=15.38$, $P=0.001)$. This was due to the fact that, for the ASD/MD group but not the ASD/NoMD group, the relationship between plasma 3NT and autism symptoms was significant $(F(1,15)=28.02, P<0.001)$. As seen in Figure $5 e$, fewer total autism symptoms (better function) were associated with higher plasma 3NT values for the ASD/MD group but not the ASD/NoMD group. No relationship was found for autism symptoms and plasma glutathione biomarkers.

Relationship between social symptoms and redox metabolism. The relationship between plasma 3NT and the SRS motivation subscale was different for the ASD/MD and ASD/NoMD groups (interaction $F(1,11)=7.20$, $P=0.02)$. This was due to the fact that, for the ASD/MD group but not the ASD/NoMD group, the relationship between plasma $3 \mathrm{NT}$ and the SRS motivation subscale was significant $(F(1,14)=11.71, P<0.01)$. As seen in Figure $5 f$, a lower SRS motivation score (better function) was associated with higher plasma 3NT values for the ASD/ MD group but not the ASD/NoMD group. In addition, plasma 3NT was found to be related to the SRS awareness subscale in a similar manner across groups $(F(1,13)=11.63$, $P=0.005)$. As seen in Figure $5 \mathrm{~g}$, a lower SRS awareness score (better function) was associated with higher plasma $3 N T$ values for both the ASD/MD and ASD/NoMD groups. No relationship was found for the SRS scale and plasma glutathione biomarkers.

Medications and supplements: differences between ASD groups. Medication and supplements were compared across groups (Table 2). A significantly greater proportion of children with the ASD/MD were receiving folate, vitamin B12, carnitine, co-enzyme Q10, B vitamins and antioxidants. 


\begin{tabular}{|c|c|c|c|c|c|}
\hline \multirow[t]{2}{*}{$\begin{array}{l}\text { Medication or } \\
\text { Supplement Class }\end{array}$} & \multicolumn{3}{|c|}{$\begin{array}{c}\text { ASD Group } \\
\% \text { Taking Medication or Supplement }\end{array}$} & \multicolumn{2}{|c|}{$\begin{array}{c}\text { Participant Age } \\
\text { Mean (SD) }\end{array}$} \\
\hline & ASD/MD & ASD/NoMD & Overall & Not Receiving & Receiving \\
\hline Stimulant & $0 \%$ & $12 \%$ & $6 \%$ & $8.4(3.3)$ & $11.3(0.2)$ \\
\hline Alpha-adrenergic & $17 \%$ & $22 \%$ & $19 \%$ & $8.6(3.4)$ & $8.2(2.4)$ \\
\hline Antipsychotic & $11 \%$ & $17 \%$ & $14 \%$ & $8.0(3.1)$ & $12.0(2.6)$ \\
\hline SSRI & $6 \%$ & $22 \%$ & $14 \%$ & $8.5(3.4)$ & $8.6(3.2)$ \\
\hline Antiepileptic & $28 \%$ & $6 \%$ & $17 \%$ & $8.7(3.6)$ & $7.8(2.0)$ \\
\hline Allergy & $33 \%$ & $22 \%$ & $28 \%$ & $8.5(3.3)$ & $8.7(3.5)$ \\
\hline Melatonin & $17 \%$ & $11 \%$ & $14 \%$ & $8.7(3.4)$ & $7.7(2.6)$ \\
\hline Fish Oil & $44 \%$ & $22 \%$ & $33 \%$ & $8.2(3.3)$ & $9.3(3.3)$ \\
\hline Folate & $72 \% *$ & $39 \%$ & $56 \%$ & $8.8(3.6)$ & $8.4(3.1)$ \\
\hline Vitamin B-12 & $50 \%{ }^{\star \star}$ & $6 \%$ & $28 \%$ & $8.1(3.4)$ & $9.5(2.9)$ \\
\hline Carnitine & $89 \%$ *** & $6 \%$ & $50 \%$ & $7.8(3.1)$ & $9.3(3.4)$ \\
\hline Co-Enzyme Q10 & $83 \%$ *** & $0 \%$ & $47 \%$ & $8.2(3.6)$ & $9.0(2.9)$ \\
\hline Other B Vitamins & $89 \%$ *** & $17 \%$ & $42 \%$ & $8.8(3.8)$ & $8.3(2.9)$ \\
\hline Other Antioxidants & $83 \%$ *** & $17 \%$ & $53 \%$ & $8.5(3.7)$ & $8.6(3.0)$ \\
\hline
\end{tabular}

Abbreviations: ASD, autism spectrum disorder; MD, mitochondrial disease; SSRI, selective serotonin reuptake inhibitors.

${ }^{\star} P<0.05 ;{ }^{* \star} P<0.01 ;{ }^{\star * \star} P<0.0001$.

Percentages represent the percentage of participants in each ASD group who were taking specific medication or supplement classes. Chi-Square was used to compare the significance of the percentages. Mean age of participants taking and not taking specific medications or supplements is listed. There was no difference in the age of patients taking or not taking medications or supplements within or between ASD groups.

The mean age for participants receiving or not receiving medications and/or supplements was not different within or between groups.

Medications and supplements: relationship to redox and inflammatory metabolism. To determine if supplements or medications provided to the participant could account for differences in redox and/or inflammatory biomarkers, we examined whether difference in biomarker values depended on whether an individual was receiving certain supplement or medication. To provide a statically valid comparison, only medications or supplements that were being given (or not given) to about half of the participants were examined. These included folate, antioxidants, carnitine, co-enzyme Q10 and $B$ vitamins. Neither 3NT, 3CT nor fGSH were influenced by the use of folate, antioxidants, carnitine, co-enzyme Q10 or B vitamins. However, participants receiving antioxidants demonstrated significantly lower GSSG (mean (s.e.), no antioxidants: $0.20 \quad(0.01)$, antioxidants: $0.18 \quad(0.01)$; $\mathrm{F}(1,16)=4.95, \quad P<0.05)$ and significantly higher $\mathrm{fGSH} /$ GSSG (mean (s.e.), no antioxidants: $8.2(0.4)$, antioxidants: $9.6(0.4) ; F(1,16)=5.69, P<0.05)$. In addition, $\mathrm{fGSH} / \mathrm{GSSG}$ was significantly higher in participants receiving co-enzyme Q10 (mean (s.e.), no co-enzyme Q10: 8.2 (0.4), co-enzyme Q10: $9.8(0.4) ; \mathrm{F}(1,16)=6.52, P<0.05)$ and/or $\mathrm{B}$ vitamins (mean (s.e.), no B vitamins: 8.2 (0.4), B vitamins: 9.6 (0.4); $\mathrm{F}(1,16)=5.82, P<0.05)$.

\section{Discussion}

In this study, development, language and behavior as well as plasma biomarkers of oxidative stress and inflammation were compared between two groups of children with ASD: those with MD (ASD/MD) and those in which MD was ruled-out (ASD/NoMD). This allowed the comparison of redox metabolism between two subgroups of children with ASD in order to determine whether abnormalities in oxidative stress are similar across all children with ASD or whether different subgroups of children with ASD may have different patterns of redox and/or inflammatory abnormalities. Overall, several plasma biomarkers of redox metabolism and inflammation differed between groups, specifically fGSH/GSSG, GSSG and $3 \mathrm{CT}$. Nonetheless, the majority of the plasma biomarkers in both ASD groups were different from a control population of typically developing children, suggesting that both ASD groups manifested abnormal redox and immune metabolism, but in slightly different ways. The difference in redox metabolism between these two ASD groups is further supported by the fact that the relationship between a measure of chronic oxidative damage, notably plasma 3NT, and many of the indices of development, language and behavior were different between the two groups. More participants in the ASD/MD group were receiving supplements that could effect redox metabolism. However, these supplements only seem to be related to differences in glutathione metabolism (that is, GSSG, GSH/GSSG), not to differences in the measure of chronic oxidative stress that was related to development, language and behavior (that is, 3NT), suggesting that receiving such supplements did not account for the difference in the relationship between development, language and behavior and chronic oxidative stress found across ASD groups. The significance of our findings is discussed further below.

A recent meta-analysis which combined the 112 available ASD/MD cases demonstrated that this subgroup of children with ASD have distinct clinical characteristics as compared with the general population of children with ASD, including a higher incidence of regression, motor delays, seizures and gastrointestinal disorders. ${ }^{11}$ The current study expands the characteristics of children with ASD/MD, demonstrating that, as compared with age- and gender-matched ASD/NoMD children, children with ASD/MD have lower adaptive behavior 
scores. Specifically, the ASD/MD group showed reduced scores in communication and daily living skills, despite having similar symptoms of autistic behavior and social dysfunction. Interestingly, the ASD/MD group also demonstrated more favorable redox metabolism, including lower concentrations of plasma GSSG and a higher fGSH/GSSG ratio, as compared with the age- and gender-matched ASD/NoMD participants. However, the higher portion of children receiving particular supplements that could affect oxidative stress could have accounted for this finding. In addition, children with ASD/MD were found to have higher elevations in the plasma biomarker for inflammation (3CT) as compared with the age- and gender-matched ASD/NoMD participants. As the vitamin supplements did not appear to be related to levels of 3CT it is less likely that this finding is a result of more participants in the ASD/MD receiving vitamin supplementation. These results suggest that children with $A S D / M D$ are true a subgroup of ASD children with distinct clinical, developmental and metabolic characteristics.

Differences in redox metabolism between the two ASD groups are particularly interesting. The ASD/MD group demonstrated a unique relationship between oxidative stress and development and behavior. A measure of chronic oxidative damage to proteins, plasma $3 \mathrm{NT}$, demonstrated the same general relationship to several indices of development and behavior in the ASD/MD children. For all three subscales on the VABS, the measure of language development, the measure of autism symptoms and a measure of social dysfunction, better skills were associated with a higher level of protein oxidation. This relationship was not observed in the ASD/noMD group. This may seems paradoxical at first, but we must consider that ROS may have several potential roles in physiology. One of the most popular interpretations in medicine is that increased ROS/ RNS represents dysfunctional metabolic systems and that an increase in ROS/RNS equates to cellular damage. Indeed, ROS has been implicated in several neurodegenerative diseases, including Alzheimer's, Parkinson's and Huntington diseases as well as Friedrich's ataxia and amyotrophic lateral sclerosis $^{69}$ and has been implicated in synaptic loss in mitochondrial encephalopathies. ${ }^{70}$ However, there are several other views on the significance of ROS in physiological systems. For example, ROS is important for long-term potentiation, a process that is essential for learning and memory, and can stimulate pathways responsible for increasing synaptic size and strength as well as synaptic vesicle recycling. ${ }^{70}$ In fact, ROS is necessary to stimulate cellular function, including mitochondrial function. ${ }^{71}$ In addition, recently the significance of ROS in ASD has been questioned. Indeed, it has been proposed that the increase in ROS associated with ASD is a cellular defense response and may not necessarily represent dysfunction redox metabolism per se. ${ }^{43}$

We believe that the positive relationship between increased plasma 3NT and cognition and behavior becomes clearer when we consider the source of ROS/RNS in individuals with mitochondrial dysfunction. ETC complexes I and III produce ROS in proportion to their activity. ${ }^{35,36}$ Dysfunctional ETC complexes may produce greater ROS and be less efficient at generating energy. ${ }^{72}$ To compensate for reduced energy production, dysfunction mitochondria may increase in number and activity. Thus, in the context of dysfunctional mitochondria, an increase in mitochondrial activity may be required to obtain near normal mitochondrial function. This increase in mitochondrial activity results in greater ROS production, especially in the context of mitochondrial dysfunction. Thus, the data presented in this study suggest that the greater the mitochondrial activity the more favorable the development (as well as a more oxidized microenvironment). This is consistent with the idea that greater mitochondrial function is associated with better development.

In contrast, the ASD/NoMD group demonstrated a more overall unfavorable pattern of glutathione metabolism relative to the ASD/MD children. The elevation in plasma GSSG without a concomitant decrease in plasma fGSH suggests that the children with ASD/NoMD are, in general, under higher extrinsic or intrinsic oxidative stress. Although such sources of oxidative stress may not be attributable to the mitochondria per se, extrinsic toxins or intrinsic sources of ROS such as inflammation are possible as are other possibilities noted above such as oxidative shielding. ${ }^{43}$ It is also possible that this effect is due to a lower number of children in the ASD/NoMD group receiving particular vitamin supplementations that can influence oxidative stress.

The biomarker of inflammation, plasma 3CT, demonstrated some interesting differences between the two ASD groups. Plasma 3CT correlated with age for the ASD/MD group but not the ASD/NoMD group such that higher plasma 3CT concentrations were found in younger ASD/MD children, as compared with ASD/NoMD children, whereas older children with ASD/MD demonstrated plasma 3CT levels that were more comparable to the children of similar age in the ASD/ NoMD group. The significance of this finding is not clear but there are several known connections between inflammation and mitochondrial dysfunction. Several reports have documented that inflammatory processes, especially involving fever, that are triggered by illnesses or vaccines, can result in neurodevelopmental regression, including regression into $A S D$, in children with underlying MD, ${ }^{73}$ even if MD was not diagnosed before the illness. ${ }^{74}$ Inflammation and inflammatory mediators can inhibit mitochondrial function ${ }^{30-32}$ and increased oxidative stress associated with inflammation may cause further mitochondrial dysfunction, ${ }^{33,34}$ potentially leading to long-term mitochondrial damage. The fact that the younger children manifested higher levels of the inflammatory marker suggests that inflammation could be a trigger that initiated the mitochondrial dysfunction and damage. Over time, the inflammation may resolve leaving the mitochondrial in a damaged state. Further research will need to examine the potential connection between mitochondrial dysfunction, MD and inflammation with respect to the development of ASD.

The strength of this study is that it has utilized two clearly differentiated groups of children with ASD. Unfortunately, because children with $M D$ are a minority of the ASD population, the number of participants in this study was limited. In addition, it is important to realize that the number of ASD subgroups is probably more than the two that have been defined and investigated in this study. Also, the sample recruited for this study, for most cases, demonstrated moderate ASD symptoms and moderate language delay, so the spectrum of ASD investigated in this study may be limited. Hopefully, future studies can capture larger populations of 
children with ASD/MD and matched ASD controls to confirm and clarify the findings of the current study and define subgroups of children with ASD/MD and ASD/NoMD. Because of the relatively limited number of children with ASD/MD, a multicenter study will most likely be necessary. In addition, as most children with MD are prescribed various vitamin supplements, which might affect mitochondrial function and redox biomarkers, future studies may target ASD children with newly diagnosed MD before starting these supplements or have the study participants wash-out the supplements for an extended period before measuring biomarkers.

This study extends our understanding of the subgroup of ASD children with MD and the variation of patterns of oxidative stress abnormalities in the ASD population. Understanding the complex interactions between redox homeostasis, inflammation and mitochondrial dysfunction is essential in order to gain a better understanding of the potentially most effective treatments for metabolic abnormalities associated with ASD. This is particularly important as evidence-based treatments for ASD are limited. Currently, there is no Food and Drug Administration-approved medical therapy that addresses either core ASD symptoms or pathophysiological processes associated with ASD. Pharmaceutical treatment that could improve core ASD symptoms as well as treat physiological abnormalities associated with ASD would provide a significant advance in the medical treatment of ASD. Currently, many treatments used for children with ASD, which address these metabolic abnormalities, particularly oxidative stress and mitochondrial dysfunction, have demonstrated efficacy in preliminary studies. ${ }^{75-77}$ Understanding which subgroups of ASD children may respond to these specific treatments will allow the development of targeted clinical treatments for ASD. In addition, the recognition and development of biomarkers, which identify ASD subgroups with specific pathophysiology, could led to identifying young children and infants who are at high risk for developing ASD during the presymptomatic period so interventions can be implemented early, potentially preventing the development of ASD.

\section{Conflict of interest}

The authors declare no conflict of interest.

Acknowledgements. This research was funded, in part, by the Jane Bostford Johnson Foundation.

1. APA. Diagnostic and Statistical Manual of Mental Disorders. 4th ed. American Psychiatric Association: Washington, DC, 1994.

2. Rice C. Prevalence of autism spectrum disorders-Autism and Developmental Disabilities Monitoring Network, United States, 2006. MMWR Surveill Summ 2009; 58: 1-20.

3. Schaefer GB, Mendelsohn NJ. Clinical genetics evaluation in identifying the etiology of autism spectrum disorders: 2013 guideline revisions. Genet Med 2013; 15: 399-407.

4. Frye RE, Rossignol DA. Mitochondrial dysfunction can connect the diverse medical symptoms associated with autism spectrum disorders. Pediatr Res 2011; 69(5 Pt 2): 41R-47RR.

5. Buie T, Campbell DB, Fuchs GJ III, Furuta GT, Levy J, Vandewater J et al. Evaluation, diagnosis, and treatment of gastrointestinal disorders in individuals with ASDs: a consensus report. Pediatrics 2010; 125(Suppl 1): S1-18

6. James SJ, Melnyk S, Jernigan S, Cleves MA, Halsted CH, Wong DH et al. Metabolic endophenotype and related genotypes are associated with oxidative stress in children with autism. Am J Med Genet B Neuropsychiatr Genet 2006; 141: 947-956.
7. Ashwood P, Krakowiak P, Hertz-Picciotto I, Hansen R, Pessah I, Van de Water J. Elevated plasma cytokines in autism spectrum disorders provide evidence of immune dysfunction and are associated with impaired behavioral outcome. Brain Behav Immun 2011; 25: 40-45.

8. Rossignol DA, Frye RE. A review of research trends in physiological abnormalities in autism spectrum disorders: immune dysregulation, inflammation, oxidative stress, mitochondrial dysfunction and environmental toxicant exposures. Mol Psychiatry 2012; 17: 389-401.

9. Ming X, Brimacombe M, Chaaban J, Zimmerman-Bier B, Wagner GC. Autism spectrum disorders: concurrent clinical disorders. J Child Neurol 2008; 23: 6-13.

10. Herbert MR. Autism: a brain disorder or a disorder that affects the brain. Clin Neuropsych 2005; 2: 354-379.

11. Rossignol DA, Frye RE. Mitochondrial dysfunction in autism spectrum disorders: a systematic review and meta-analysis. Mol Psychiatry 2012; 17: 290-314.

12. Frye RE. Biomarker of abnormal energy metabolism in children with autism spectrum disorder. N Am J Med Sci 2012; 5: 141-147.

13. Giulivi $\mathrm{C}$, Zhang YF, Omanska-Klusek A, Ross-Inta C, Wong S, Hertz-Picciotto I et al. Mitochondrial dysfunction in autism. JAMA 2010; 304: 2389-2396.

14. Hallmayer J, Cleveland S, Torres A, Phillips J, Cohen B, Torigoe T et al. Genetic heritability and shared environmental factors among twin pairs with autism. Arch Gen Psychiatry 2011; 68: 1095-1102.

15. Frye RE, Naviaux RK. Autistic disorder with complex IV overactivity: a new mitochondrial syndrome. J Ped Neurol 2011; 9: 427-434.

16. Graf WD, Marin-Garcia J, Gao HG, Pizzo S, Naviaux RK, Markusic D et al. Autism associated with the mitochondrial DNA G8363A transfer RNA ${ }^{\text {Lys }}$ mutation. J Child Neurol 2000; 15: 357-361.

17. Frye RE. Novel cytochrome b gene mutations causing mitochondrial disease in autism. $J$ Ped Neurol 2011; 10: 35-40.

18. Herbert MR, Russo JP, Yang S, Roohi J, Blaxill M, Kahler SG et al. Autism and environmental genomics. Neurotoxicology 2006; 27: 671-684.

19. Ashwood P, Van de Water J. Is autism an autoimmune disease? Autoimmun Rev 2004; 3 : 557-562.

20. Bayona-Bafaluy MP, Lopez-Gallardo E, Montoya J, Ruiz-Pesini E. Maternally inherited susceptibility to cancer. Biochim Biophys Acta 2011; 1807: 643-649.

21. Nishigaki Y, Fuku N, Tanaka M. Mitochondrial haplogroups associated with lifestyle-related diseases and longevity in the Japanese population. Geriatr Gerontol Int 2010; 10(Suppl 1): S221-S235.

22. Fowler BA, Woods JS. Ultrastructural and biochemical changes in renal mitochondria during chronic oral methyl mercury exposure: the relationship to renal function. Exp $\mathrm{Mol}$ Pathol 1977; 27: 403-412.

23. Shenker BJ, Guo TL, O I, Shapiro IM. Induction of apoptosis in human T-cells by methyl mercury: temporal relationship between mitochondrial dysfunction and loss of reductive reserve. Toxicol Appl Pharmacol 1999; 157: 23-35.

24. Goyer RA. Toxic and essential metal interactions. Annu Rev Nutr 1997; 17: 37-50

25. Pourahmad J, Mihajlovic A, O'Brien PJ. Hepatocyte lysis induced by environmental metal toxins may involve apoptotic death signals initiated by mitochondrial injury. Adv Exp Med Biol 2001; 500: 249-252.

26. Hiura TS, Li N, Kaplan R, Horwitz M, Seagrave JC, Nel AE. The role of a mitochondria pathway in the induction of apoptosis by chemicals extracted from diesel exhaust particles. J Immunol 2000; 165: 2703-2711.

27. Wong PW, Garcia EF, Pessah IN. ortho-substituted PCB95 alters intracellular calcium signaling and causes cellular acidification in PC12 cells by an immunophilin-dependent mechanism. J Neurochem 2001; 76: 450-463.

28. Sherer TB, Richardson JR, Testa CM, Seo BB, Panov AV, Yagi T et al. Mechanism of toxicity of pesticides acting at complex I: relevance to environmental etiologies of Parkinson's disease. J Neurochem 2007; 100: 1469-1479.

29. Yamano T, Morita S. Effects of pesticides on isolated rat hepatocytes, mitochondria, and microsomes II. Arch Environ Contam Toxicol 1995; 28: 1-7.

30. Samavati L, Lee I, Mathes I, Lottspeich F, Huttemann M. Tumor necrosis factor alpha inhibits oxidative phosphorylation through tyrosine phosphorylation at subunit I of cytochrome c oxidase. J Biol Chem 2008; 283: 21134-21144.

31. Vempati UD, Diaz F, Barrientos A, Narisawa S, Mian AM, Millan JL et al. Role of cytochrome $C$ in apoptosis: increased sensitivity to tumor necrosis factor alpha is associated with respiratory defects but not with lack of cytochrome $\mathrm{C}$ release. $\mathrm{Mol} \mathrm{Cell} \mathrm{Biol}$ 2007; 27: 1771-1783

32. Suematsu N, Tsutsui $H$, Wen J, Kang D, Ikeuchi M, Ide T et al. Oxidative stress mediates tumor necrosis factor-alpha-induced mitochondrial DNA damage and dysfunction in cardiac myocytes. Circulation 2003; 107: 1418-1423.

33. Vali S, Mythri RB, Jagatha B, Padiadpu J, Ramanujan KS, Andersen JK et al. Integrating glutathione metabolism and mitochondrial dysfunction with implications for Parkinson's disease: a dynamic model. Neuroscience 2007; 149: 917-930.

34. Fernandez-Checa JC, Kaplowitz N, Garcia-Ruiz C, Colell A, Miranda M, Mari M et al. GSH transport in mitochondria: defense against TNF-induced oxidative stress and alcoholinduced defect. Am J Physiol 1997; 273(1 Pt 1): G7-G17.

35. Koopman WJ, Nijtmans LG, Dieteren CE, Roestenberg P, Valsecchi F, Smeitink JA et al. Mammalian mitochondrial complex I: biogenesis, regulation, and reactive oxygen species generation. Antioxid Redox Signal 2010; 12: 1431-1470. 
36. Distelmaier F, Koopman WJ, van den Heuvel LP, Rodenburg RJ, Mayatepek E, Willems PH et al. Mitochondrial complex I deficiency: from organelle dysfunction to clinical disease. Brain 2009; 132(Pt 4): 833-842.

37. Chinta SJ, Andersen JK. Nitrosylation and nitration of mitochondrial complex I in Parkinson's disease. Free Radic Res 2011; 45: 53-58.

38. Wiesner RJ, Zsurka G, Kunz WS. Mitochondrial DNA damage and the aging process: facts and imaginations. Free Radic Res 2006; 40: 1284-1294.

39. Ghanizadeh A, Akhondzadeh S, Hormozi M, Makarem A, Abotorabi-Zarchi M, Firoozabadi A. Glutathione-related factors and oxidative stress in autism, a review. Current medicinal chemistry 2012; 19: 4000-4005.

40. Naviaux RK. Oxidative shielding or oxidative stress? J Pharmacol Exp Ther 2012; 342 : 608-618.

41. Frustaci A, Neri M, Cesario A, Adams JB, Domenici E, Dalla Bernardina B et al. Oxidative stress-related biomarkers in autism: systematic review and meta-analyses. Free Radic Biol Med 2012; 52: 2128-2141.

42. Deth R, Muratore C, Benzecry J, Power-Charnitsky VA, Waly M. How environmental and genetic factors combine to cause autism: a redox/methylation hypothesis. Neurotoxicology 2008; 29: 190-201.

43. James SJ, Rose S, Melnyk S, Jernigan S, Blossom S, Pavliv O et al. Cellular and mitochondrial glutathione redox imbalance in lymphoblastoid cells derived from children with autism. FASEB J 2009; 23: 2374-2383.

44. Melnyk S, Fuchs GJ, Schulz E, Lopez M, Kahler SG, Fussell JJ et al. Metabolic imbalance associated with methylation dysregulation and oxidative damage in children with autism. J Autism Dev Disord 2012; 42: 367-377.

45. Rose S, Melnyk S, Pavliv O, Bai S, Nick TG, Frye RE et al. Evidence of oxidative damage and inflammation associated with low glutathione redox status in the autism brain. Transl Psychiatry 2012; 2: e134.

46. Chauhan A, Audhya T, Chauhan V. Brain region-specific glutathione redox imbalance in autism. Neurochem Res 2012; 37: 1681-1689.

47. Napoli E, Wong S, Giulivi C. Evidence of reactive oxygen species-mediated damage to mitochondrial DNA in children with typical autism. Mol Autism 2013; 4: 2.

48. Damodaran LP, Arumugam G. Urinary oxidative stress markers in children with autism. Redox Rep 2011; 16: 216-222.

49. Ali A, Waly MI, Al-Farsi YM, Essa MM, Al-Sharbati MM, Deth RC. Hyperhomocysteinemia among Omani autistic children: a case-control study. Acta Biochim Pol 2011; 58: 547-551.

50. Waly MI, Hornig M, Trivedi M, Hodgson N, Kini R, Ohta A et al. Prenatal and postnatal epigenetic programming: implications for Gl, immune, and neuronal function in autism. Autism Res Treat 2012; 2012: 190930.

51. James SJ, Shpyleva S, Melnyk S, Pavliv O, Pogribny IP. Complex epigenetic regulation of Engrailed-2 (EN-2) homeobox gene in the autism cerebellum. Transl Psychiatry 2013; 3: e232.

52. Finkelstein JD. Metabolic regulatory properties of S-adenosylmethionine and S-adenosylhomocysteine. Clin Chem Lab Med 2007; 45: 1694-1699.

53. Miller AL. The methylation, neurotransmitter, and antioxidant connections between folate and depression. Altern Med Rev 2008; 13: 216-226.

54. Mitochondrial Medicine Society's Committee on DHaas RH, Parikh S, Falk MJ, Saneto RP, Wolf NI, Darin N et al. The in-depth evaluation of suspected mitochondrial disease. Mol Genet Metab 2008; 94: 16-37.

55. Munnich A, Rustin P. Clinical spectrum and diagnosis of mitochondrial disorders. Am J Med Genet 2001; 106: 4-17.

56. Wong LJ. Pathogenic mitochondrial DNA mutations in protein-coding genes. Muscle Nerve 2007; 36: 279-293.

57. Wong LJ, Cobb BR, Chen TJ. Molecular analysis of mitochondrial DNA point mutations by polymerase chain reaction. Methods Mol Biol 2006; 336: 135-143.
58. Bai RK, Perng CL, Hsu CH, Wong LJ. Quantitative PCR analysis of mitochondrial DNA content in patients with mitochondrial disease. Ann N Y Acad Sci 2004; 1011: 304-309.

59. Bai RK, Wong LJ. Simultaneous detection and quantification of mitochondrial DNA deletion(s), depletion, and over-replication in patients with mitochondrial disease. $\mathrm{J} \mathrm{Mol}$ Diagn 2005; 7: 613-622.

60. Kirby DM, Thorburn DR, Turnbull DM, Taylor RW. Biochemical assays of respiratory chain complex activity. Methods Cell Biol 2007; 80: 93-119.

61. Roe CR, Roe DS. Recent developments in the investigation of inherited metabolic disorders using cultured human cells. Mol Genet Metab 1999; 68: 243-257.

62. Morava E, van den Heuvel L, Hol F, de Vries MC, Hogeveen M, Rodenburg RJ et al. Mitochondrial disease criteria: diagnostic applications in children. Neurology 2006; 67: 1823-1826.

63. Sparrow S, Cicchetti D, Balla D. Vineland Adaptive Behavior Scales. 2nd edn Minneapolis, MN, 2005.

64. Zimmerman IL, Steiner VG, Pond RE. Preschool Language Scale. 4th edn Harcourt Assessment: San Antonio, TX, 2002.

65. Brownell R. Expressive One-Word Picture Vocabulary Test Manual. Academic Therapy Publications: Novato, CA, 2000.

66. Constantino JN. The Social Responsiveness Scale. Western Psychological Services: Los Angeles, 2002.

67. Melnyk S, Pogribna M, Pogribny I, Hine RJ, James SJ. A new HPLC method for the simultaneous determination of oxidized and reduced plasma aminothiols using coulometric electrochemical detection. J Nutr Biochem 1999; 10: 490-497.

68. Laird NM, Ware JH. Random-effects models for longitudinal data. Biometrics 1982; 38 : 963-974.

69. Emerit J, Edeas M, Bricaire F. Neurodegenerative diseases and oxidative stress. Biomed Pharmacother 2004; 58: 39-46.

70. Milton VJ, Sweeney ST. Oxidative stress in synapse development and function. Dev Neurobiol 2012; 72: 100-110.

71. Ristow M, Schmeisser S. Extending life span by increasing oxidative stress. Free Radic Biol Med 2011; 51: 327-336.

72. Fato R, Bergamini C, Leoni S, Strocchi P, Lenaz G. Generation of reactive oxygen species by mitochondrial complex I: implications in neurodegeneration. Neurochem Res 2008; 33 : 2487-2501.

73. Edmonds JL, Kirse DJ, Kearns D, Deutsch R, Spruijt L, Naviaux RK. The otolaryngological manifestations of mitochondrial disease and the risk of neurodegeneration with infection. Arch Otolaryngol Head Neck Surg 2002; 128: 355-362.

74. Shoffner J, Hyams L, Langley GN, Cossette S, Mylacraine L, Dale J et al. Fever plus mitochondrial disease could be risk factors for autistic regression. J Child Neurol 2010; 25. 429-434.

75. Frye RE, Rossignol DA. Treatments for mitochondrial dysfunction associated with autism spectrum disorders. J Ped Biochem 2012; 2: 241-249.

76. Lofthouse N, Hendren R, Hurt E, Arnold LE, Butter E. A review of complementary and alternative treatments for autism spectrum disorders. Autism Res Treat 2012; 2012: 870391.

77. Rossignol DA. Novel and emerging treatments for autism spectrum disorders: a systematic review. Ann Clin Psychiatry 2009; 21: 213-236.

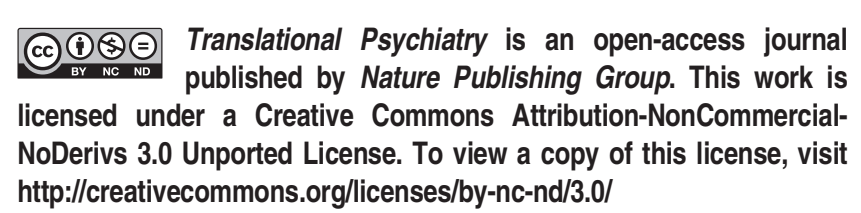

